injured body part was the head (48\%) with concussions (40\%). Final model revealed sex, age, position during contact, contact surface, and play legality were significantly associated with HNF injuries. Controlling for play legality and position during contact, U18-men injured during contact with an opposing player had the highest probability of HNF injuries (51\%) and a higher probability than U18 women $(P=0.004)$. Meanwhile, women 18-24 $(P=0.019)$ and over $30 \quad(P=0.042)$ who were injured during contact with the ground had a higher probability of HNF injuries than men.

Conclusions Under-18 male players involved in contact with players were most at risk for HNF injuries. Meanwhile, adult women 18-24 and 30-years old had a higher probability of sustaining a HNF injury when injured during contact with the ground. Tackle techniques, break falls, and other interactions employed by developing women and men players, including tackles or collisions, should be reviewed in detail for injury reduction.

\section{THE NORDIC HAMSTRING EXERCISE: AWARENESS, IMPLEMENTATION AND OPINION OF WORLDWIDE ATHLETES}

\begin{abstract}
1,2,3 Wesam Saleh A Al Attar, ${ }^{1}$ Amirah M Akkam, ${ }^{1}$ Nada N Al Hazmi, ${ }^{1}$ Eman F Aljedaani, ${ }^{4}$ Hussain Saleh H Ghulam, ${ }^{5}$ Hend A Dorgham, ${ }^{3}$ Ross H Sanders. 'Department of Physical Therapy, Faculty of Applied Medical Science, Umm Al Qura University, Makkah, Saudi Arabia; '2Department of Sport, Exercise and Health, Faculty of Medicine, University of Basel, Basel, Switzerland; ${ }^{3}$ Discipline of Exercise and Sport Science, Faculty of Medicine and Health, The University of Sydney, Sydney, Australia; ${ }^{4}$ Department of Rehabilitation Medical Sciences, Faculty of Applied Medical Science, Najran University, Najran, Saudi Arabia; ${ }^{5}$ Department of Physical Therapy, Ras Eltein General Hospital, Alexandria, Egypt
\end{abstract}

\subsection{6/bjsports-2021-IOC. 140}

Background The Nordic hamstring exercise is an effective program in reducing the hamstring injuries, which is one of the most common non-contact lower limb injuries, especially in sports requiring acceleration, maximal sprints, and sudden change in direction of running, as sprinting, soccer, and rugby. Objective To assess the awareness, implementation, and opinion of the worldwide athletes about the Nordic hamstring exercise in preventing hamstring injury.

Design A cross-sectional study.

Setting An online survey for different sports.

Patients (or Participants) A total of 1142 male and female athletes from different sports (American football, athletics, badminton, baseball, basketball, bodybuilding, boxing, cricket, CrossFit, cycling, soccer, gymnastics, handball, ice hockey, ice skating, judo, ju-jitsu, karate, korfball, lacrosse, modern pentathlon, netball, rugby league, rugby union, running, skateboarding, skiing, snowboarding, softball, squash, swimming, table tennis, taekwondo, tchoukball, tennis, trampolining, volleyball, weightlifting and wrestling) completed the survey.

Interventions (or Assessment of Risk Factors) The questionnaire consisted of questions relating to the awareness level, implementation rate and opinion of the Nordic hamstring exercise.

Main Outcome Measurements The primary outcomes were awareness level, implementation rate, and opinion of the effectiveness of the Nordic hamstring exercise in hamstring injury.
Results A total of $641(56 \%)$ of the athletes were aware of the Nordic hamstring exercise, 519 (45.4\%) were implementing the Nordic hamstring exercise in their current practice. Athletes who implemented the Nordic hamstring exercise reported a positive opinion about the program efficacy, with a score of $8.4 \pm 1$ out of 10 .

Conclusions Many athletes were aware of the Nordic hamstring exercise and implementing it in their training routine, as they found it effective in decreasing the hamstring injury rates. However, further work needs to be done to educate athletes about the importance of implementing the exercise and its effectiveness in preventing hamstring injury to enhance implementation.

\section{THE NORDIC HAMSTRING EXERCISE: AWARENESS, IMPLEMENTATION AND OPINION OF WORLDWIDE PROFESSIONAL AND SEMI-PROFESSIONAL SOCCER PLAYERS AND COACHES}

1,3Wesam Saleh A Al Attar, ${ }^{1}$ Ramy G Komir, '1 Ibrahim A Bukhari, 'Ali A Alzubeadi, ${ }^{4}$ Hussain Saleh H Ghulam, ${ }^{3}$ Ross H Sanders. 'Department of Physical Therapy, Faculty of Applied Medical Science, Umm Al Qura University, Makkah, Saudi Arabia; ${ }^{2}$ Department of Sport, Exercise and Health, Faculty of Medicine, University of Basel, Basel, Switzerland; ${ }^{3}$ Discipline of Exercise and Sport Science, Faculty of Medicine and Health, The University of Sydney, Sydney, Australia; ${ }^{4}$ Department of Rehabilitation Medical Sciences, Faculty of Applied Medical Science, Najran University, Najran, Saudi Arabia

\subsection{6/bjsports-2021-IOC.141}

Background Soccer has high injury risk, especially for hamstring muscle injuries, and the Nordic hamstring exercise is a well-known intervention to prevent them.

Objective To assess the professional and semi-professional soccer players and coaches' awareness, implementation, and opinion of the Nordic hamstring exercise.

Design A cross-sectional study.

Setting An online survey for all continental football federations.

Patients (or Participants) A total of 812 male (88.3\%) and female (11.7\%) professional and semi-professional soccer players and coaches completed the survey.

Interventions (or Assessment of Risk Factors) The questionnaire consisted of questions relating to the awareness level, implementation rate, and opinion of the of the Nordic hamstring exercise. Questions development was guided by several authors whose expertise is in sport medicine and injury prevention.

Main Outcome Measurements The primary outcomes were awareness level, implementation rate, and opinion of the effectiveness of the Nordic hamstring exercise in reducing hamstring injury.

Results A total of 395 (48.6\%) of professional and semi-professional soccer players and coaches were aware of the Nordic hamstring exercise, $355(43.7 \%)$ were implementing the Nordic hamstring exercise in their current practice. Participants who implemented the Nordic hamstring exercise reported a positive opinion about the program efficacy, with a score of $8.6 \pm 1$ out of 10 .

Conclusions Further work needs to be done to educate soccer players and coaches about the importance of implementing the Nordic hamstring exercise and its effectiveness in preventing hamstring injury to enhance implementation of the Nordic hamstring exercise. 